PROCEEDINGS OF THE

AMERICAN MATHEMATICAL SOCIETY

Volume 140, Number 9, September 2012, Pages 3133-3143

S 0002-9939(2012)11145-1

Article electronically published on January 5, 2012

\title{
PRESCRIBED COMPRESSIONS OF DUAL HYPERCYCLIC OPERATORS
}

\author{
KIT C. CHAN \\ (Communicated by Richard Rochberg)
}

\begin{abstract}
If $M$ is a closed subspace of a separable, infinite dimensional Hilbert space $H$ with $\operatorname{dim}(H / M)=\infty$, then we show that every bounded linear operator $A: M \rightarrow M$ is the compression of a dual hypercyclic operator $T: H \rightarrow H$.
\end{abstract}

On a separable, infinite dimensional Banach space $X$, a bounded linear operator $T: X \rightarrow X$ is said to be hypercyclic on $X$ if there is a vector $x \in X$ whose orbit $\operatorname{orb}(T, x)=\left\{x, T x, T^{2} x, T^{3} x, \ldots\right\}$ is dense in $X$. Such a vector $x$ is called a hypercyclic vector for $T$. When the operator $T: X \rightarrow X$ is hypercyclic on $X$ and its adjoint operator $T^{*}: X^{*} \rightarrow X^{*}$ is hypercyclic on the dual space $X^{*}$ of $X$, then we say that $T$ is dual hypercyclic. Since an orbit is a countable set, dual hypercyclicity can only take place when both $X$ and $X^{*}$ are separable. However, $X$ is separable whenever its dual $X^{*}$ is, but the converse is not always true.

Separability does not present an issue when the Banach space $X$ is indeed a Hilbert space $H$, because the adjoint $T^{*}$ is a bounded linear operator on $H$ itself. In fact, it was the Hilbert space setting that the concept of dual hypercyclicity started to develop. A fundamental question is whether dual hypercyclic operators on $H$ can ever exist. This question was originally raised by Herrero 4. The first example of such an operator was found by Salas [6]. Later he [7] provided another example using a general result for hypercyclic bilateral weighted shift operators.

Recently generalizations of dual hypercyclic operators to a Banach space $X$ were studied. For instance, Petersson [5] showed that any infinite dimensional Banach space $X$ with a shrinking symmetric basis, such as $c_{0}$ and $\ell^{p}$ with $1<p<\infty$, admits a dual hypercyclic operator $T: X \rightarrow X$. Then Salas 8 showed that any Banach space $X$ with a separable dual space $X^{*}$ admits a dual hypercyclic operator. More recently, Shkarin [10] studied dual hypercyclic tuples of operators on Banach spaces, and Salas 9 studied dual disjoint hypercyclic operators.

In the present paper we return to the setting of a separable, infinite dimensional Hilbert space $H$ and study compressions of dual hypercyclic operators $T: H \rightarrow H$, making use of unique Hilbert space properties. Our main result is Theorem 2 below, which states that the compression of a dual hypercyclic operator $T$ onto a closed subspace $M$ of infinite codimension in $H$ can coincide with any prescribed operator $A$ on $M$. In other words, if $P: H \rightarrow H$ is the orthogonal projection onto a closed subspace $M$ with $\operatorname{dim}(H / M)=\infty$, then for any bounded linear

Received by the editors March 21, 2011.

2010 Mathematics Subject Classification. Primary 47A16, 47A20; Secondary 47A05.

Key words and phrases. Adjoint operator, dual hypercyclic operator, compression.

(C)2012 American Mathematical Society Reverts to public domain 28 years from publication 
operator $A: M \rightarrow M$, there is a dual hypercyclic operator $T: H \rightarrow H$ such that $\left.P T P\right|_{M}=A$. Compressions of a hypercyclic operator were studied by Salas [7], who showed that if $T: H \rightarrow H$ is a hypercyclic operator and $N$ is an invariant subspace of its adjoint $T^{*}$, then the compression of $T$ to $N$ is hypercyclic on $N$.

To prove Theorem 2, we additively decompose the Hilbert space $H$ as an orthogonal sum of infinitely many copies of a nonzero closed subspace $M$ with $\operatorname{dim}(H / M)=$ $\infty$; that is, $H=\bigoplus_{j=-\infty}^{\infty} M_{j}$, where each $M_{j}$ is a closed subspace of $H$ that is isomorphic to $M$ and $M_{i} \perp M_{j}$ whenever $i \neq j$. We identify the original closed subspace $M$ with $M_{0}$ in $H$, and the prescribed operator $A: M \rightarrow M$ becomes an operator $A: M_{0} \rightarrow M_{0}$. By extending $A$ so that $\left.A\right|_{M_{0}^{\perp}}=0$, we can view $A$ as a bounded linear operator $A: H \rightarrow H$. Assume $\operatorname{dim} M=\infty$, and let $\left\{e_{j, 1}, e_{j, 2}, e_{j, 3}, \ldots\right\}$ be an orthonormal basis of $M_{j}$. Hence $e_{j, i} \perp e_{m, n}$ whenever $(j, i) \neq(m, n)$. Let $S: H \rightarrow H$ be the unitary operator given by $S e_{j, i}=e_{j+1, i}$ whenever $i, j \in \mathbb{Z}$. In fact, $\left.S\right|_{M_{j}}$ is an isomorphism between $M_{j}$ and $M_{j+1}$. Lastly, we need a number $a>\max \{1,\|A\|\}$ to help set up the following technical lemma.

Lemma 1. Suppose $k$ is a positive integer, and for each integer $j$ with $|j| \leq k$ we are given a positive weight $w_{j}$ satisfying $0<w_{j} \leq a$ and also vectors $x_{j}$ and $y_{j}$ in $M_{j}$. Let $\epsilon>0$. Then there exists an integer $n \geq 2 k+2$, and there exist weights $w_{j}$ with $0<w_{j} \leq a$ and also vectors $x_{j} \in M_{j}$ for all integers $j$ with $1+k \leq|j| \leq n+k$ such that if $D$ is defined by

$$
D e_{j, i}= \begin{cases}w_{j} e_{j-1, i} & \text { whenever } i \in \mathbb{Z} \text { and }|j| \leq n+k, \\ 0 & \text { otherwise, }\end{cases}
$$

then

(i)

$\left\|(D+A)^{n}\left(\sum_{i=-k}^{n-k-1} x_{i}\right)\right\|<\epsilon$,

(ii) $(D+A)^{n}\left(\sum_{i=n-k}^{n+k} x_{i}\right)=\sum_{i=-k}^{k} y_{i}$,

(iii) $\sum_{i=1+k}^{n+k}\left\|x_{i}\right\|^{2}<\epsilon$, and

(iv) $x_{i}=0$ whenever $-n-k \leq i \leq-1-k$.

Proof. Before we determine a desired integer $n$ for the definition of $D$ in (10), we first observe that if $j \neq 0$, then

$$
\left.(D+A)\right|_{M_{j}}=\left.D\right|_{M_{j}} .
$$

We also observe that if $m$ is an integer with $1+k \leq m \leq n+k$, then for any vector $x_{m} \in M_{m}$, (11) gives

$$
(D+A)^{m}\left(x_{m}+\sum_{i=-k}^{k} x_{i}\right) \perp M_{j} \quad \text { whenever } j \geq 1 .
$$


Note that $w_{j}$ are given positive numbers whenever $|j| \leq k$. We now define $w_{j}=a$ for all indices $j$ with $1+k \leq j \leq m$. If we let $P: H \rightarrow H$ be the orthogonal projection onto $M_{0}$, then we trivially have

$$
P(D+A)^{m}\left(\sum_{i=-k}^{k} x_{i}\right) \in M_{0} .
$$

Furthermore, if we specifically take the vector $x_{m}$ in $M_{m}$ to be

$$
x_{m}=\frac{-1}{w_{1} \cdots w_{k} w_{k+1} \cdots w_{m}} S^{m} P(D+A)^{m}\left(\sum_{i=-k}^{k} x_{i}\right),
$$

then we can take $P(D+A)^{m}$ on both sides of the above equation to obtain

$$
P(D+A)^{m}\left(\sum_{i=-k}^{k} x_{i}\right)=-P(D+A)^{m} x_{m}
$$

By combining with (2), we have

$$
(D+A)^{m}\left(x_{m}+\sum_{i=-k}^{k} x_{i}\right) \perp M_{j} \quad \text { whenever } j \geq 0 .
$$

Recall that $w_{k+1}=\cdots=w_{m}=a>\|A\|$ and $\|S\|=1$, and note that $x_{m}$ can be rewritten as

$$
x_{m}=\frac{-1}{w_{1} \cdots w_{k} w_{k+1} \cdots w_{m}} S^{m} P A^{m-k}(D+A)^{k}\left(\sum_{i=-k}^{k} x_{i}\right) .
$$

Thus we can first choose a large enough integer $m$, even before we determine the desired integer $n$ for the definition of $D$, so that the above formulation of $x_{m}$ gives

$$
\left\|x_{m}\right\|^{2}<\frac{\epsilon}{2} .
$$

Then we determine a large enough integer $n$ with $n \geq m+k+1 \geq 2 k+2$, so that if we define $w_{-1-k}=\cdots=w_{-n-k}=a^{-1}<1$, then it follows from (11) and (3) that

$$
\left\|(D+A)^{n}\left(x_{m}+\sum_{i=-k}^{k} x_{i}\right)\right\|<\epsilon .
$$

Thus if we define $x_{1+k}=\cdots=x_{m-1}=0$ and $x_{m+1}=\cdots=x_{n-k-1}=0$, then (i) is clearly satisfied.

We now check how (ii) can be satisfied whenever $n \geq 2 k+2$ by appropriately choosing $x_{n-k}, \ldots, x_{n+k}$. For that, let $w_{m+1}=\cdots=w_{n+k}=a$ to complete the definitions for $w_{j}$ in the whole range $1+k \leq|j| \leq n+k$ as required by the lemma. For the given vectors $y_{j}$ in $M_{j}$ with $|j| \leq k$ as in the statement of our lemma, we first let

$$
x_{n-k}=\frac{1}{w_{1-k} \cdots w_{n-k}} S^{n} y_{-k} .
$$

Next, for every index $i$ with $1-k \leq i \leq 0$, let

$$
x_{n+i}=\frac{1}{w_{1+i} \cdots w_{n+i}}\left(S^{n} y_{i}-\frac{1}{w_{i}} S^{n+i} A S^{1-i} y_{i-1}\right) .
$$


Lastly, for every index $i$ with $1 \leq i \leq k$, let

$$
x_{n+i}=\frac{1}{w_{1+i} \cdots w_{n+i}} S^{n} y_{i} .
$$

One can easily verify that the above definitions give $x_{i} \in M_{i}$ whenever $n-k \leq i \leq$ $n+k$.

To establish (ii), we first make an observation that if $1-k \leq i \leq 0$, then

$$
\begin{aligned}
& (D+A)^{n}\left(\frac{S^{n} y_{i-1}}{w_{i} \cdots w_{n+i-1}}-\frac{S^{n+i} A S^{1-i} y_{i-1}}{w_{i} \cdots w_{n+i-1} w_{n+i}}\right) \\
= & (D+A)^{-i+1}\left(\frac{(D+A)^{n+i-1} S^{n} y_{i-1}}{w_{i} \cdots w_{n+i-1}}-\frac{(D+A)^{n+i-1} S^{n+i} A S^{1-i} y_{i-1}}{w_{i} \cdots w_{n+i-1} w_{n+i}}\right) .
\end{aligned}
$$

To continue our computations, we note that $S^{n} y_{i-1} \in M_{n+i-1}$ and $S^{n+i} A S^{1-i} y_{i-1}$ $\in M_{n+i}$. Thus we can simplify the above expression as

$$
(D+A)^{-i+1}\left(\frac{S^{1-i} y_{i-1}}{w_{i} \cdots w_{0}}-\frac{S A S^{1-i} y_{i-1}}{w_{i} \cdots w_{0} w_{1}}\right)
$$

Since $S^{1-i} y_{i-1} \in M_{0}$ and $S A S^{1-i} y_{i-1} \in M_{1}$, we can further reduce the above expression to

$$
\begin{aligned}
& (D+A)^{-i}\left(\left(\frac{S^{-i} y_{i-1}}{w_{i} \cdots w_{-1}}+\frac{A S^{1-i} y_{i-1}}{w_{i} \cdots w_{0}}\right)-\frac{A S^{1-i} y_{i-1}}{w_{i} \cdots w_{0}}\right) \\
= & (D+A)^{-i}\left(\frac{S^{-i} y_{i-1}}{w_{i} \cdots w_{-1}}\right) \\
= & y_{i-1} .
\end{aligned}
$$

Hence we conclude from our above observation that if $1-k \leq i \leq 0$, then

$$
(D+A)^{n}\left(\frac{S^{n} y_{i-1}}{w_{i} \cdots w_{n+i-1}}-\frac{S^{n+i} A S^{1-i} y_{i-1}}{w_{i} \cdots w_{n+i-1} w_{n+i}}\right)=y_{i-1}
$$

We are now ready to establish (ii) by first using the definitions of $x_{i}$ in (5), (6), and (7):

$$
\begin{aligned}
(D+A)^{n} & \left(\sum_{i=n-k}^{n+k} x_{i}\right) \\
= & (D+A)^{n}\left(\frac{S^{n} y_{-k}}{w_{1-k} \cdots w_{n-k}}+\right. \\
& \left.+\sum_{i=1-k}^{0} \frac{1}{w_{1+i} \cdots w_{n+i}}\left(S^{n} y_{i}-\frac{1}{w_{i}} S^{n+i} A S^{1-i} y_{i-1}\right)+\sum_{i=1}^{k} x_{n+i}\right),
\end{aligned}
$$


which can be rearranged as

$$
\begin{aligned}
& \quad(D+A)^{n}\left(\sum_{i=1-k}^{0}\left(\frac{S^{n} y_{i-1}}{w_{i} \cdots w_{n+i-1}}-\frac{S^{n+i} A S^{1-i} y_{i-1}}{w_{i} \cdots w_{n+i}}\right)\right) \\
& +(D+A)^{n}\left(\frac{S^{n} y_{0}}{w_{1} \cdots w_{n}}\right)+(D+A)^{n}\left(\sum_{i=1}^{k} \frac{S^{n} y_{i}}{w_{1+i} \cdots w_{n+i}}\right) \\
& =\sum_{i=1-k}^{0} y_{i-1}+y_{0}+\sum_{i=1}^{k} y_{i}, \text { by (마) and the fact that } s^{n} y_{i} \in M_{n+i} .
\end{aligned}
$$

Hence we have established (ii).

Since we have set $w_{m+1}=\cdots=w_{n+k}=a>1$, and $S$ is an isometry, we can assume that $n$ is chosen large enough so that the definitions for $x_{n-k}, \ldots, x_{n+k}$ in (5), (6), (7) give

$$
\sum_{i=n-k}^{n+k}\left\|x_{i}\right\|^{2}<\frac{\epsilon}{2} .
$$

This inequality, along with (4) and the fact that $x_{1+k}=\cdots=x_{m-1}=x_{m+1}=$ $\cdots=x_{n-k-1}=0$, yields (iii). The proof of the lemma is completed by setting $x_{-1-k}=\cdots=x_{-n-k}=0$ to establish (iv).

For an arbitrary operator $A: M_{0} \rightarrow M_{0}$, the above lemma provides an operator $D$ in (1) that shifts some orthonormal basis members in the backward direction, that is, taking some $e_{j, i}$ to $w_{j} e_{j-1, i}$. For the adjoint operator $A^{*}: M_{0} \rightarrow M_{0}$, one can easily modify the techniques in the lemma to provide an operator $D^{\prime}$ which shifts in the forward direction, taking some $e_{j, i}$ to $w_{j+1} e_{j+1, i}$. We omit the statement of the forward shifting version of the lemma.

To prove our main result in Theorem 2 below, we apply the lemma to $A$ and then apply the forward shifting version of the lemma to $A^{*}$. The two applications to $A$ and $A^{*}$ are then repeated inductively.

Theorem 2. Let $H$ be a separable, infinite dimensional Hilbert space and let $M$ be a closed subspace of $H$ with $\operatorname{dim}(H / M)=\infty$. Let $P: H \rightarrow H$ be the orthogonal projection onto $M$. If $A: M \rightarrow M$ is a bounded linear operator, then there is an operator $T: H \rightarrow H$ such that

(1) $T$ is dual hypercyclic,

(2) $\left.P T P\right|_{M}=A$, and

(3) $\left.P T^{*} P\right|_{M}=A^{*}$.

Proof. Since $M$ has infinite codimension in $H$, we can use an orthonormal basis argument to additively decompose $H$ as an orthogonal sum $H=\bigoplus_{j=-\infty}^{\infty} M_{j}$, where $M_{0}$ is the closed subspace $M$ given in the statement of our theorem and each $M_{j}$ is isomorphic to $M$.

In the rest of the proof, we assume that $\operatorname{dim} M=\infty$, and the same argument works if $\operatorname{dim} M$ is finite. For that, let $\left\{e_{j, 1}, e_{j, 2}, e_{j, 3}, \ldots\right\}$ be an orthonormal basis of $M_{j}$. Since $A$ takes $M_{0}$ to $M_{0}$, we can view $A$ as an operator from $H$ to $H$ with $\left.A\right|_{M_{0}^{\perp}}=0$. It follows that $\overline{\operatorname{ran} A^{*}}=(\operatorname{ker} A)^{\perp} \subset M_{0}$ and also that $M_{0}^{\perp} \subset(\operatorname{ran} A)^{\perp}=$ $\operatorname{ker} A^{*}$, and so we can also view the original adjoint operator $A^{*}: M_{0} \rightarrow M_{0}$ as 
an operator from $H$ to $H$ with $\left.A^{*}\right|_{M_{0}^{\perp}}=0$. That allows us to define an operator $T: H \rightarrow H$ by $T=A+B$ and so $T^{*}=A^{*}+B^{*}$, where $B: H \rightarrow H$ is a linear map defined by $B e_{j, i}=w_{j} e_{j-1, i}$ for all integers $i, j$ and $\left\{w_{j}: j \in \mathbb{Z}\right\}$ is a bounded two-sided sequence of positive numbers. Thus $B$ takes each $M_{j}$ to $M_{j-1}$ and $\left.B\right|_{M_{j}}$ is in the form of " $w_{j}$ times a Hilbert space isomorphism". In fact, if $S: H \rightarrow H$ is the unitary operator given by $S e_{j, i}=e_{j+1, i}$ whenever $i, j \in \mathbb{Z}$, then $\left.S\right|_{M_{j}}$ is an isomorphism from $M_{j}$ to $M_{j+1}$, and $\left.B\right|_{M_{j}}=\left.w_{j} S^{-1}\right|_{M_{j}}$. It is easy to verify that $\left.B^{*}\right|_{M_{j}}=\left.w_{j+1} S\right|_{M_{j}}$; that is, $B^{*} e_{j, i}=w_{j+1} e_{j+1, i}$. In addition, the definition of $T$ gives $\left.P T P\right|_{M_{0}}=A$ and $\left.P T^{*} P\right|_{M_{0}}=A^{*}$. In fact, $B$ is a bilateral backward shift, and $\|B\|=\sup \left|w_{j}\right|<\infty$. In the rest of the proof we need to choose $w_{j}$ so that $T$ and $T^{*}$ are hypercyclic.

Let $a$ be a number with $a>\max \{1,\|A\|\}$, and let $v_{1}, v_{2}, v_{3}, \ldots$ be an enumeration of all vectors, each of which has only a finite number of nonzero coefficients $\left\langle v, e_{j, i}\right\rangle$ and all coefficients are rationals. The set of all $v_{i}$ is dense in $H$. Let $k_{1} \geq 1$ be such that $v_{1} \in \bigoplus_{|j| \leq k_{1}} M_{j}$. After choosing $k_{1}$, let $w_{j}=a$ and $x_{j}=0$ whenever $|j| \leq k_{1}$, and write $v_{1}=\sum_{|j| \leq k_{1}} y_{j}$ with each $y_{j} \in M_{j}$. Let $\epsilon_{1}=\left(2 a^{2 k_{1}}\right)^{-1}>0$. The lemma provides an integer $n_{1}$ and weights $w_{j}$ with $0<w_{j} \leq a$ and vectors $x_{j} \in M_{j}$ whenever $1+k_{1} \leq|j| \leq n_{1}+k_{1}$ such that if $D_{1}$ is given by

$$
D_{1} e_{j, i}= \begin{cases}w_{j} e_{j-1, i} & \text { whenever } i \in \mathbb{Z} \text { and }|j| \leq n_{1}+k_{1}, \\ 0 & \text { otherwise, }\end{cases}
$$

then statements (i), (ii), (iii), and (iv) are satisfied.

With the above setting, we apply the lemma to the operator $A^{*}$ in a direction opposite to the statement of the lemma, that is, to obtain a forward shift operator $D^{\prime}$. Hence, for the same vector $v_{1}=\sum_{|j| \leq k_{1}} y_{j}$ we can continue to define $w_{j}$ and $x_{j}$ by setting $k_{1}^{\prime}=n_{1}+k_{1}$ and $\epsilon_{1}^{\prime}=\left(2 a^{2 k_{1}^{\prime}}\right)^{-1}>0$. The lemma provides an integer $n_{1}^{\prime}$ and weights $w_{j}$ with $0<w_{j} \leq a$, and vectors $x_{j} \in M_{j}$, for all indices $j$ in the range $1+k_{1}^{\prime} \leq|j| \leq n_{1}^{\prime}+k_{1}^{\prime}$. Since we are applying the statement of the lemma in the opposite direction, statement (iv) gives $x_{i}=0$ for those indices $i$ in the range $1+k_{1}^{\prime} \leq i \leq n_{1}^{\prime}+k_{1}^{\prime}$, and statement (iii) gives

$$
\sum_{i=-n_{1}^{\prime}-k_{1}^{\prime}}^{-1-k_{1}^{\prime}}\left\|x_{i}\right\|^{2}<\frac{1}{2 a^{2 k_{1}^{\prime}}}
$$

In addition, the lemma provides a forward shift operator $D_{1}^{\prime}$ by

$$
D_{1}^{\prime} e_{j, i}= \begin{cases}w_{j+1} e_{j+1, i} & \text { if } i \in \mathbb{Z} \text { and }-n_{1}^{\prime}-k_{1}^{\prime} \leq j \leq n_{1}^{\prime}+k_{1}^{\prime}-1 \\ 0 & \text { otherwise }\end{cases}
$$

such that statements (i) and (ii) become

$$
\left\|\left(D_{1}^{\prime}+A^{*}\right)^{n_{1}^{\prime}}\left(\sum_{i=-n_{1}^{\prime}+k_{1}^{\prime}+1}^{k_{1}^{\prime}} x_{i}\right)\right\|<\epsilon_{1}^{\prime}
$$

and

$$
\left(D_{1}^{\prime}+A^{*}\right)^{n_{1}^{\prime}}\left(\sum_{i=-n_{1}^{\prime}-k_{1}^{\prime}}^{-n_{1}^{\prime}+k_{1}^{\prime}} x_{i}\right)=v_{1}
$$


Note that the lemma provides the term $w_{-n_{1}^{\prime}-k_{1}^{\prime}}$ that we do not actually use in the above definition of $D_{1}^{\prime}$, and this term causes no consequence in our subsequent argument. In the second step, we let $k_{2}=n_{1}^{\prime}+k_{1}^{\prime}$, and $\epsilon_{2}=\left(2^{2} a^{2 k_{2}}\right)^{-1}>0$ and assume that, without loss of generality, $v_{2} \in \bigoplus_{|j| \leq k_{2}} M_{j}$. In the case that $v_{2}$ is not in the subspace $\bigoplus_{|j| \leq k_{2}} M_{j}$, we can choose $v_{i}$ with the least integer $i$ such that $v_{i}$ is in that subspace. We can use the lemma to define operator $D_{2}$ and then $D_{2}^{\prime}$ for the same vector $v_{2}$, as we define $D_{1}$ and $D_{1}^{\prime}$ in the previous case for $v_{1}$.

Inductively, in the $m$-th step we take $k_{m}=n_{m-1}^{\prime}+k_{m-1}^{\prime}$ and $\epsilon_{m}=\left(2^{m} a^{2 k_{m}}\right)^{-1}>$ 0 and we can assume, without loss of generality, that $v_{m} \in \bigoplus_{|j| \leq k_{m}} M_{j}$. Together with those $w_{j}$ and $x_{j}$ already defined for indices $j$ with $|j| \leq n_{m-1}^{\prime}+k_{m-1}^{\prime}=k_{m}$ in the $(m-1)$-th step, we obtain from the lemma an integer $n_{m}$ and weights $w_{j}$ with $0<w_{j} \leq a$, and vectors $x_{j}$ in $M_{j}$, where $|j| \leq n_{m}+k_{m}$ and in fact,

$$
x_{i}=0 \text { for those indices } i \text { in the range }-n_{m}-k_{m} \leq i \leq-1-k_{m},
$$

such that if $D_{m}$ is given by

$$
D_{m} e_{j, i}= \begin{cases}w_{j} e_{j-1, i} & \text { whenever } i \in \mathbb{Z} \text { and }|j| \leq n_{m}+k_{m}, \\ 0 & \text { otherwise }\end{cases}
$$

then

$$
\sum_{i=1+k_{m}}^{n_{m}+k_{m}}\left\|x_{i}\right\|^{2}<\frac{1}{2^{m} a^{2 k_{m}}}
$$

and

$$
\left\|\left(D_{m}+A\right)^{n_{m}}\left(\sum_{i=-k_{m}}^{n_{m}-k_{m}-1} x_{i}\right)\right\|<\frac{1}{2^{m} a^{2 k_{m}}}
$$

and also

$$
\left(D_{m}+A\right)^{n_{m}}\left(\sum_{i=n_{m}-k_{m}}^{n_{m}+k_{m}} x_{i}\right)=v_{m}
$$

Next we apply the lemma to obtain a forward shift $D_{m}^{\prime}$ for the same vector $v_{m}$, but for the adjoint $A^{*}$ instead of $A$ itself. For that purpose, let $k_{m}^{\prime}=n_{m}+k_{m}$ and $\epsilon_{m}^{\prime}=\left(2^{m} a^{2 k_{m}^{\prime}}\right)^{-1}>0$. Together with those $w_{j}$ and $x_{j}$ that have already been defined for indices $j$ in the range $-k_{m}^{\prime} \leq j \leq k_{m}^{\prime}$, we obtain from the lemma an integer $n_{m}^{\prime}$ and weights $w_{j}$ with $0<w_{j} \leq a$ and vectors $x_{j}$ in $M_{j}$ whenever $|j| \leq n_{m}^{\prime}+k_{m}^{\prime}$, and in fact

$$
x_{i}=0 \text { for those indices } i \text { in the range } 1+k_{m}^{\prime} \leq i \leq n_{m}^{\prime}+k_{m}^{\prime},
$$

such that

$$
\sum_{i=-n_{m}^{\prime}-k_{m}^{\prime}}^{-1-k_{m}^{\prime}}\left\|x_{i}\right\|^{2}<\frac{1}{2^{m} a^{2 k_{m}^{\prime}}}
$$

In addition, the lemma provides an operator $D_{m}^{\prime}$ given by

$$
D_{m}^{\prime} e_{j, i}= \begin{cases}w_{j+1} e_{j+1, i} & \text { if } i \in \mathbb{Z} \text { and }-n_{m}^{\prime}-k_{m}^{\prime} \leq j \leq n_{m}^{\prime}+k_{m}^{\prime}-1 \\ 0 & \text { otherwise }\end{cases}
$$


such that

$$
\left\|\left(D_{m}^{\prime}+A^{*}\right)^{n_{m}^{\prime}}\left(\sum_{i=-n_{m}^{\prime}+k_{m}^{\prime}+1}^{k_{m}^{\prime}} x_{i}\right)\right\|<\epsilon_{m}^{\prime}
$$

and also

$$
\left(D_{m}^{\prime}+A^{*}\right)^{n_{m}^{\prime}}\left(\sum_{i=-n_{m}^{\prime}-k_{m}^{\prime}}^{-n_{m}^{\prime}+k_{m}^{\prime}} x_{i}\right)=v_{m} .
$$

With all weights $w_{j}$ given by the inductive process above, we define an operator $B: H \rightarrow H$ by $\left.B\right|_{M_{j}}=\left.w_{j} S^{-1}\right|_{M_{j}}$ as we have outlined in the beginning of this proof. The operator $B$ is bounded because $0<w_{j} \leq a$. Also, with all vectors $x_{j}$ given by the inductive process, we let $x=\sum_{j \in \mathbb{Z}} x_{j}$, which represents a vector in $H$ because the terms $x_{j}$ are mutually orthogonal and square summable. To verify that, we note that $k_{m}^{\prime}=n_{m}+k_{m}$, and $k_{m+1}=n_{m}^{\prime}+k_{m}^{\prime}$ by their definitions, and hence

$$
\begin{aligned}
& \sum_{i=1+k_{1}}^{\infty}\left\|x_{i}\right\|^{2} \\
= & \sum_{m=1}^{\infty}\left(\sum_{i=1+k_{m}}^{n_{m}+k_{m}}\left\|x_{i}\right\|^{2}+\sum_{i=1+k_{m}^{\prime}}^{n_{m}^{\prime}+k_{m}^{\prime}}\left\|x_{i}\right\|^{2}\right) \\
= & \sum_{m=1}^{\infty}\left(\sum_{i=1+k_{m}}^{n_{m}+k_{m}}\left\|x_{i}\right\|^{2}\right) \text { by (13), }
\end{aligned}
$$

and hence by (10) we have

$$
\sum_{i=1+k_{1}}^{\infty}\left\|x_{i}\right\|^{2}<\sum_{m=1}^{\infty} \frac{1}{2^{m} a^{2 k_{m}}}<\infty .
$$

Similarly, using (9) and (14), we obtain

$$
\sum_{i=-\infty}^{-k_{1}^{\prime}-1}\left\|x_{i}\right\|^{2}<\infty
$$

and so the sum $\sum_{j \in \mathbb{Z}} x_{j}$ defines a vector in $H$.

We now proceed to prove that if we write $x=\sum_{j \in \mathbb{Z}} x_{j}$, then $x$ is a hypercyclic vector for both $A+B$ and $A^{*}+B^{*}$. We begin by rewriting (12) as

$$
(A+B)^{n_{m}}\left(\sum_{i=n_{m}-k_{m}}^{n_{m}+k_{m}} x_{i}\right)=v_{m},
$$

and hence by orthogonality we have

$$
\begin{aligned}
& \left\|(A+B)^{n_{m}} x-v_{m}\right\|^{2} \\
= & \left\|(A+B)^{n_{m}}\left(\sum_{i=-\infty}^{n_{m}-k_{m}-1} x_{i}\right)\right\|^{2}+\left\|(A+B)^{n_{m}}\left(\sum_{i=n_{m}+k_{m}+1}^{\infty} x_{i}\right)\right\|^{2} .
\end{aligned}
$$


We continue our computations with the two summands in (17) separately. For the first summand we note that by orthogonality, we can write it as the sum of two terms:

$$
\left\|(A+B)^{n_{m}}\left(\sum_{i=-n_{m}-k_{m}}^{n_{m}-k_{m}-1} x_{i}\right)\right\|^{2}+\left\|B^{n_{m}}\left(\sum_{i=-\infty}^{-n_{m}-k_{m}-1} x_{i}\right)\right\|^{2}
$$

To continue our estimations, note that (9) and (11) give

$$
\begin{aligned}
& \left\|(A+B)^{n_{m}}\left(\sum_{i=-n_{m}-k_{m}}^{n_{m}-k_{m}-1} x_{i}\right)\right\|^{2} \\
= & \left\|(A+B)^{n_{m}}\left(\sum_{i=-k_{m}}^{n_{m}-k_{m}-1} x_{i}\right)\right\|^{2} \\
< & \left(\frac{1}{2^{m} a^{2 k_{m}}}\right)^{2} .
\end{aligned}
$$

Thus we obtain an estimate for the first summand in (17) using (9) and (18) as follows:

$$
\left\|(A+B)^{n_{m}}\left(\sum_{i=-\infty}^{n_{m}-k_{m}-1} x_{i}\right)\right\|^{2}<\left(\frac{1}{2^{m} a^{2 k_{m}}}\right)^{2}+a^{2 n_{m}} \sum_{j=m}^{\infty} \sum_{i=-k_{j+1}}^{-n_{j}-k_{j}-1}\left\|x_{i}\right\|^{2} .
$$

Note that $k_{j}^{\prime}=n_{j}+k_{j}$ and $k_{j+1}=n_{j}^{\prime}+k_{j}^{\prime}$, and so

$$
\begin{aligned}
a^{2 n_{m}} \sum_{j=m}^{\infty} \sum_{i=-k_{j+1}}^{-n_{j}-k_{j}-1}\left\|x_{i}\right\|^{2} & =a^{2 n_{m}} \sum_{j=m}^{\infty}\left(\sum_{i=-n_{j}^{\prime}-k_{j}^{\prime}}^{-1-k_{j}^{\prime}}\left\|x_{i}\right\|^{2}\right) \\
& <a^{2 n_{m}} \sum_{j=m}^{\infty} \frac{1}{2^{j} a^{2 k_{j}^{\prime}}}, \text { by (14) } \\
& \left.<\sum_{j=m}^{\infty} \frac{1}{2^{j}}, \text { (because } k_{j}^{\prime} \geq k_{m}^{\prime}=n_{m}+k_{m}\right) \\
& =2^{1-m} .
\end{aligned}
$$

Hence the first summand in (17) is bounded above by $\left(2^{m} a^{2 k_{m}}\right)^{-2}+2^{1-m}$, which goes to 0 as $m$ goes to $\infty$. 
To estimate the second summand in (17) we first use (13) to obtain that $x_{i}=0$ for those indices $i$ in the range $1+k_{j}^{\prime}=n_{j}+k_{j}+1 \leq i \leq n_{j}^{\prime}+k_{j}^{\prime}=k_{j+1}$, and hence

$$
\begin{aligned}
& \left\|(A+B)^{n_{m}}\left(\sum_{i=n_{m}+k_{m}+1}^{\infty} x_{i}\right)\right\|^{2} \\
= & \left\|B^{n_{m}}\left(\sum_{j=m}^{\infty} \sum_{i=1+k_{j+1}}^{n_{j+1}+k_{j+1}} x_{i}\right)\right\|^{2} \\
\leq & \|B\|^{2 n_{m}}\left(\sum_{j=m}^{\infty} \sum_{i=1+k_{j+1}}^{n_{j+1}+k_{j+1}}\left\|x_{i}\right\|^{2}\right) \\
< & a^{2 n_{m}} \sum_{j=m}^{\infty}\left(\frac{1}{2^{j+1} a^{2 k_{j+1}}}\right) \text { by (10) } \\
\leq & \left.\sum_{j=m}^{\infty} \frac{1}{2^{j+1}} \quad \text { because } k_{j+1} \geq k_{m+1}>k_{m}^{\prime}>n_{m}\right) \\
= & 2^{-m},
\end{aligned}
$$

which goes to 0 as $m$ goes to $\infty$. Since $T=A+B$, it follows from (17) that $\left\|T^{n_{m}} x-v_{m}\right\|^{2} \rightarrow 0$ as $m \rightarrow \infty$. Recall that the set $\left\{v_{1}, v_{2}, v_{3}, \ldots\right\}$ is dense in $H$, and so is the set $\left\{x, T x, T^{2} x, \ldots\right\}$. In other words, $T$ is hypercyclic.

A similar argument using (9), (10), (13), (14), (15), and (16) shows that $T^{*}=$ $A^{*}+B^{*}$ is also hypercyclic. Thus $T$ is dual hypercyclic.

In the above proof, we construct a vector $x$ that is hypercyclic for both $A+B$ and $A^{*}+B^{*}$. As a matter of fact, for any two hypercyclic operators $T_{1}$ and $T_{2}$ on $H$, there must be a vector that is hypercyclic for both operators because for any hypercyclic operator $T$, the set of all hypercyclic vectors $H C(T)$ is a dense $G_{\delta}$ set. By the Baire Category Theorem, $H C\left(T_{1}\right) \cap H C\left(T_{2}\right)$ is also a dense $G_{\delta}$ set.

In the above theorem, if we take $M$ to be a one dimensional subspace and $A: M \rightarrow M$ to be the zero operator, then the proof provides a bilateral weighted shift operator that is dual hypercyclic. This result was obtained by Salas 7 .

It may be a surprising fact that dual hypercyclic operators can exist on a separable, infinite dimensional Hilbert space $H$, but Theorem 2 shows that they are indeed very common in terms of prescribed behaviors of their compressions on a closed subspace $M$ with infinite codimension in $H$. For such subspaces $M$, Chan and Turcu [2] and Grivaux [3] showed that any bounded linear operator $A: M \rightarrow M$ can be extended to a chaotic operator $T: H \rightarrow H$. Here, a chaotic operator is a hypercyclic operator with a dense set of periodic points $x$; that is, $T^{n} x=x$ for some positive integer $n$ depending on $x$. However, a chaotic operator cannot be dual hypercyclic, because if $T$ has a periodic point, say $T^{n} x=x$, then for any vector $y$ in $H$ the set $\left\{\left\langle T^{* k n} y, x\right\rangle \mid k \geq 0\right\}$ cannot be dense in the scalar field, and hence $T^{* n}$ cannot have a hypercyclic vector. That contradicts Ansari's result [1] that the $n$-th power of a hypercyclic operator is also hypercyclic. 


\section{REFERENCES}

[1] S. I. Ansari, Hypercyclic and cyclic vectors, Journal of Functional Analysis, 128 (1995), 374-383. MR:1319961 (96h:47002)

[2] K. C. Chan and G. Turcu, Chaotic extensions of operators on Hilbert subspaces, Revista de la Real Academia de Ciencias Exactas, Físicas y Naturales, Serie A. Matemáticas, 105 (2011), 415-421.

[3] S. Grivaux, Topologically transitive extensions of bounded operators, Math Z., 249 (2005), 85-96. MR2106971 (2005g:47008)

[4] D. A. Herrero, Limits of hypercyclic and supercyclic operators, Journal of Functional Analysis, 99 (1991), 179-190. MR.1120920 (92g:47026)

[5] H. Petersson, Spaces that admit hypercyclic operators with hypercyclic adjoints, Proceedings of the American Mathematical Society, 134 (2006), 1671-1676. MR2204278 (2007b:47020)

[6] H. Salas, A hypercyclic operator whose adjoint is also hypercyclic, Proceedings of the American Mathematical Society, 112 (1991), 765-770. MR.1049848 (91j:47016)

[7] H. Salas, Hypercyclic weighted shifts, Transactions of the American Mathematical Society, 347 (1995), 993-1004. MR1249890 (95e:47042)

[8] H. Salas, Banach spaces with separable duals support dual hypercyclic operators, Glasgow Mathematical Journal, 49 (2007), 281-290. MR2347261 (2008j:47008)

[9] H. Salas, Dual disjoint hypercyclic operators, J. Math. Anal. Appl., 374 (2011), 106-117. MR2726191

[10] S. Shkarin, A short proof of existence of disjoint hypercyclic operators, Journal of Mathematical Analysis and Applications, 367 (2010), 713-715, MR2607296 (2011f:47009)

Department of Mathematics and Statistics, Bowling Green State University, Bowling Green, OHio 43403

E-mail address: kchan@bgsu.edu 